\title{
Fibre-induced drag reduction
}

\author{
J. J. J. GILLISSEN ${ }^{1}$, B. J. BOERSMA ${ }^{1}$, P. H. MORTENSEN ${ }^{2}$ \\ AND H. I. ANDERSSON \\ ${ }^{1}$ Laboratory for Aero and Hydrodynamics, J.M. Burgers Center, Leeghwaterstraat 21, \\ 2628 CA Delft, The Netherlands \\ ${ }^{2}$ Department of Energy and Process Engineering, Norwegian University of Science and \\ Technology, 7491 Trondheim, Norway
}

(Received 27 October 2007 and in revised form 14 February 2008)

We use direct numerical simulation to study turbulent drag reduction by rigid polymer additives, referred to as fibres. The simulations agree with experimental data from the literature in terms of friction factor dependence on Reynolds number and fibre concentration. An expression for drag reduction is derived by adopting the concept of the elastic layer.

\section{Introduction}

The addition of long-chain linear polymers to turbulent pipe flows induces a reduction of the friction drag. Similar effects have also been observed for other additives, such as surfactants, macroscopic slender particles and gas bubbles. For an overview see Gyr \& Bewersdorff (1995). The drag reduction phenomenon is exploited in a number of applications, including the Trans Alaska Pipeline. Besides economical motivations, many studies on drag reduction have been performed to gain understanding of wallbounded turbulence and rheology of polymeric liquids, such as Virk (1971), Lumley (1973), Ryskin (1987), Sreenivasan \& White (2000) and Benzi et al. (2005).

Virk (1971) found that friction data in polymer-solution pipe flow can be rationalized by assuming the following parameterization for the velocity profile:

$$
\bar{u}^{+}= \begin{cases}y^{+} & \text {if } 0<y^{+}<11.6 \\ 11.7 \log y^{+}-17 & \text { if } 11.6<y^{+}<11.6+\delta_{E}^{+} \\ 2.5 \log y^{+}+9.2 \log \left(11.6+\delta_{E}^{+}\right)-17 & \text { if } 11.6+\delta_{E}^{+}<y^{+}<R e_{\tau} / 2 .\end{cases}
$$

Variables with superscript + are given in wall units, i.e. the velocity $\bar{u}^{+}=\bar{u} / U_{\tau}$ and distance to the wall $y^{+}=y U_{\tau} / \nu$ are scaled with solvent kinematic viscosity $v$ and friction velocity $U_{\tau}=(-(\mathrm{d} \bar{\Pi} / \mathrm{d} x)(D / \rho)(1 / 4))^{1 / 2}$, where $-\mathrm{d} \bar{\Pi} / \mathrm{d} x$ is the pressure gradient which drives the flow, $D$ is the pipe diameter and $\rho$ is the fluid mass density. The non-dimensional pipe diameter is referred to as the frictional Reynolds number $R e_{\tau}=U_{\tau} D / v$, which is related to the bulk Reynolds number $R e=u_{b} D / v$ and the friction factor $f=U_{\tau}^{2} / u_{b}^{2}$ by

$$
R e_{\tau}=\sqrt{f} R e .
$$

From the pipe wall $\left(y^{+}=0\right)$ to the pipe centreline $\left(y^{+}=R e_{\tau} / 2\right)$, equation (1.1) describes three layers, referred to as the viscous layer with thickness 11.6, the elastic layer with thickness $\delta_{E}^{+}$and the inertial layer, respectively. Without polymer additives $\delta_{E}^{+}=0$, equation (1.1) reduces to the Newtonian law of the wall (Tennekes \& Lumley 

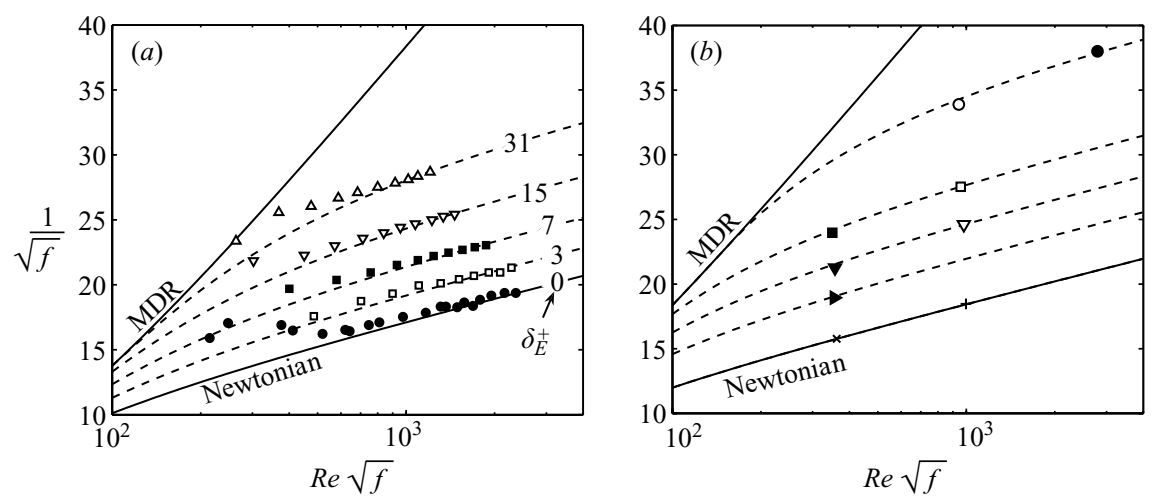

FIGURE 1. (a) Friction factor versus Reynolds number in drag-reduced pipe flow. Lines, model ((1.1) and (1.3)); symbols, measurements using Xanthan solutions with different mass fraction $c_{m}$ (Sasaki 1991a): $\bullet, c_{m}=0 ; \square, c_{m}=10 \times 10^{-6} ; \mathbf{\square}, c_{m}=25 \times 10^{-6} ; \nabla, c_{m}=50 \times 10^{-6} ; \triangle$, $c_{m}=100 \times 10^{-6}$. (b) Friction factor versus Reynolds number in drag-reduced channel flow. Lines, model ((1.1) and (4.1)); symbols, simulations with parameters listed in table 1, below.

1973). With polymer additives $\delta_{E}^{+}>0$ and the profile in the inertial layer is parallel shifted upward compared to the Newtonian profile. The friction factor $f$ is related to the non-dimensional velocity, averaged over the pipe cross-sectional area:

$$
\frac{1}{\sqrt{f}}=\frac{8}{R e_{\tau}^{2}} \int_{0}^{R e_{\tau} / 2}\left(R e_{\tau} / 2-y^{+}\right) \bar{u}^{+} \mathrm{d} y^{+} .
$$

A parameterization for $f$ in terms of $R e$ and $\delta_{E}^{+}$is obtained by inserting (1.1) into (1.3). Figure 1 $(a)$ shows this relation for fixed values of $\delta_{E}^{+}$in Prandtl-Kármán (PK) coordinates (Tennekes \& Lumley 1973). For $R e_{\tau} \rightarrow \infty$, the drag reduction curves $\left(\delta_{E}^{+}>0\right)$ are parallel shifted upward compared to the Newtonian curve $\left(\delta_{E}^{+}=0\right)$.

An important polymer property is flexibility, describing its ability to coil and stretch. In this context polymers can be categorized into flexible and rigid, each having very different drag reduction characteristics (Virk 1975). In contrast to flexible polymers, for which an experimentally based theory has been formulated (Virk 1971), rigid ones, which we refer to as fibres, have been studied far less extensively and an equivalent theory is lacking.

The present work aims at providing a basis for such theory by means of direct numerical simulation (DNS). Before turning to the simulations, we first review the experimentally established knowledge. Figure 1(a) shows experimental data in PKcoordinates (Sasaki 1991a). Results are shown for different fibre mass fractions $c_{m}$, defined as the mass of the fibres per unit mass of the solution. For sufficiently large $R e_{\tau}$ the data for each $c_{m}$ follow lines of constant $\delta_{E}^{+}$. The friction data appears to be parameterized with (1.1) and (1.3), and $\delta_{E}^{+}$therein is independent of $R e_{\tau}$. In the limit $R e_{\tau} \rightarrow \infty$ we can write the following relation between friction factor and Reynolds number:

$$
\frac{1}{\sqrt{f}}=2.5 \log \operatorname{Re} \sqrt{f}+9.2 \log \left(1+0.086 \delta_{E}^{+}\right) .
$$

This typical behaviour of constant $\delta_{E}^{+}$as a function of $R e_{\tau}$ distinguishes fibres from flexible polymers. The configuration of a flexible polymer varies from randomly coiled to fully stretched with increasing fluid deformation, introducing a rather complex Reynolds number dependence in their drag reduction efficiency (Virk 1971). 

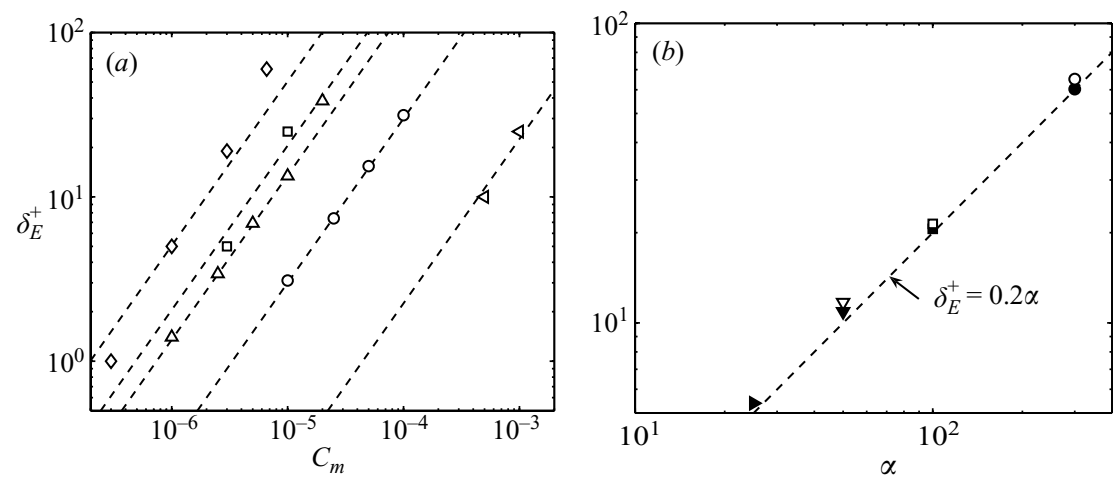

FigURE 2. (a) Elastic layer thickness versus mass fraction in pipe flow experiments. $\bigcirc$, Xanthan (Sasaki 1991a); $\triangle$, Na-polyacrylate (Sasaki 1991b); $\triangleleft$, Xanthan (Bewersdorff \& Singh 1988); $\square$, partially hydrolyzed polyacrylamide (Virk 1975); $\diamond$, partially hydrolyzed polyacrylamide (Virk et al. 1997). The dashed lines have slope 1. (b) Elastic layer versus concentration parameter in channel flow simulations. Symbols correspond to simulation parameters listed in table 1 , below.

A complete description of the friction factor in fibre solutions requires a relation between $\delta_{E}^{+}$and properties of the fibres. Here we consider the dependence on the fibre mass fraction $c_{m}$. Figure 2(a) shows $\delta_{E}^{+}$as a function of $c_{m}$ for various fibre solutions in $\log$-log coordinates. Each $\delta_{E}^{+}$-value is obtained by fitting (1.4) to a set of measured data points on the PK-plane. There is an approximately linear dependence of $\delta_{E}^{+}$on $c_{m}$, demonstrated by the dashed lines which are drawn with slope 1 . There seems to be a very simple empirical law, that the drag reduction parameter $\delta_{E}^{+}$is proportional to the number of fibres in the flow. As can be seen from figure $2(a)$, the different solutions yield different values for $\delta_{E}^{+} / c_{m}$, indicating a further dependence of $\delta_{E}^{+}$on the hydraulic properties of the fibres. To date, this dependence remains unknown.

This work is aimed at closing this problem, by means of DNS. In contrast to experiments, all conditions in simulations are known, which is of key importance for interpreting polymeric turbulent flows. However, owing to computer restrictions a limited level of physical detail can be taken into account. Therefore we take care to determine the physical significance of the simulations by comparing them to the available experimental data. To our knowledge this study is the first to quantitatively compare DNS of drag reduction to experiments. The limited detail, on the other hand, also has advantages. The reduced equations contain only the most important parameters and so a parametric study can yield a relation for drag reduction in terms of the Reynolds number and fibre properties. This would have been more difficult or even impossible if irrelevant side effects were taken into account.

\section{Mathematical model}

\subsection{Assumptions}

We use DNS to study the friction factor in turbulent fibre-solution channel flow. The choice of the channel geometry instead of pipe is motivated by the fact that the dynamics in the near-wall layer, responsible for the friction factor, are similar in pipe and channel flow (Tennekes \& Lumley 1973), but the problems related to the singularity in the cylindrical coordinate system do not have to be addressed in Cartesian calculations. 
The effect of the fibres on the fluid mechanics is modelled by adding the fibre stress to the fluid equations of motion. The equations governing this stress are based on simplifying assumptions. Here we will compare these assumptions to the experimental conditions of Sasaki (1991b). The fluid used in these experiments consists of Na-polyacrylate with molecular weight $M_{w}=1.7 \mathrm{Mg} \mathrm{mol}^{-1}$ dissolved in distilled water at room temperature $T=3 \times 10^{2} \mathrm{~K}, \rho=10^{3} \mathrm{~kg} \mathrm{~m}^{-3}$ and $v=10^{-6} \mathrm{~m}^{2} \mathrm{~s}^{-1}$. This polymer can be regarded a neutrally buoyant, rigid particle of length $l \approx 5 \times 10^{-6} \mathrm{~m}$ and diameter $d \sim 10^{-9} \mathrm{~m}$. The fibre aspect ratio $r=l / d \sim 10^{4}$ is large enough that the effects of a finite aspect ratio can be ignored (Manhart 2003). Other relevant experimental parameters are the frictional Reynolds number $R e_{\tau} \sim 10^{3}$, the pipe diameter $D=6 \times 10^{-3} \mathrm{~m}$ and the mass fraction $c_{m} \sim 10^{-5}$. Brownian motion can be neglected since the rotary diffusion time scale $t_{B} \approx \rho \nu l^{3} /\left(10 k_{B} T\right)=3 \mathrm{~s}$ (Doi \& Edwards $1986)$ is much larger than the large-eddy turnover time $t_{L} \approx\left(D^{2} / \nu\right) R e_{\tau}^{-1}=4 \times 10^{-2} \mathrm{~s}$ (Tennekes \& Lumley 1973). Here $k_{B}$ is the Boltzmann constant. The flow field around an individual fibre can be assumed to be a linear function of space since fibre length $l \approx 5 \times 10^{-6} \mathrm{~m}$ is smaller than the Kolmogorov length scale $l_{K} \approx D R e_{\tau}^{-3 / 4}=3 \times 10^{-5} \mathrm{~m}$ (Tennekes \& Lumley 1973). Fibre inertia is neglected since the particle response time $t_{p} \approx d^{2} / \nu=1 \times 10^{-12} \mathrm{~s}$ (Mortensen et al. 2008) is much smaller than the Kolmogorov time scale $t_{K} \approx\left(D^{2} / v\right) R e_{\tau}^{-3 / 2}=1 \times 10^{-3}$ s (Tennekes \& Lumley 1973).

Because of computer restrictions, we ignore hydrodynamic and physical interactions between fibres: an individual fibre moves and interacts with the flow as if the solution were dilute (Doi \& Edwards 1986). Ignoring these events allows a statistical description, which is numerically tractable. Since in principle drag reduction does not occur in dilute solutions, this assumption is not satisfied under realistic conditions. Consequently, the physical significance of our numerical model is questionable and will therefore be determined by a comparison to experimental data from the literature.

\subsection{Governing equations}

Under the above assumptions, the flow is governed by the incompressible NavierStokes equations, supplemented by the divergence of the fibre stress tensor $\boldsymbol{\tau}$ (Doi \& Edwards 1986):

$$
\begin{gathered}
\rho \frac{\mathrm{D} \boldsymbol{u}}{\mathrm{D} t}=\nabla \cdot(-\Pi \boldsymbol{\delta}+2 \mu \boldsymbol{S}+\boldsymbol{\tau}), \\
\nabla \cdot \boldsymbol{u}=0, \\
\boldsymbol{\tau}=2 \alpha \mu \boldsymbol{S}:\langle\boldsymbol{p p p p}\rangle .
\end{gathered}
$$

Here $\boldsymbol{u}$ is the fluid velocity vector, $t$ is time, $\nabla$ is the gradient operator, $\delta$ is the unit tensor, $\mathrm{D} / \mathrm{D} t=\partial / \partial t+\boldsymbol{u} \cdot \nabla$ is the material derivative, $\boldsymbol{S}=\frac{1}{2}\left(\nabla \boldsymbol{u}^{T}+\nabla \boldsymbol{u}\right)$ is the rate-of-strain tensor, $\Pi$ is the pressure, $\mu=v \rho$ is the solvent dynamic viscosity and $\boldsymbol{p}$ is the fibre orientation unit vector. The concentration parameter $\alpha$ is related to the fibre number density $n$ and fibre length $l$ as:

$$
\alpha \approx 0.1 n l^{3} \text {. }
$$

For simplicity we have neglected the logarithmic dependence of the numerical factor 0.1 on the fibre aspect ratio. Parameter $\alpha$ is the ratio of the scalar magnitude of the fibre stress and the Newtonian stress, and therefore measures the non-Newtonian character of the flow. To estimate a realistic value for $\alpha$ we use $n=c_{m} \rho N_{A} / M_{w}$, where $N_{A}$ is the Avogadro number. For the experimental conditions described in $\S 2.1$ we find $\alpha \approx 44$. 
The fibre stress involves averaging $\langle\cdots\rangle$ over a statistical ensemble of fibres, contained in a (small) volume, centred at the point where the stress is to be determined. The fibre stress $\tau$ can be interpreted as a viscous stress, with viscosity depending on the orientation of the fibres relative to the axis of fluid deformation. The required information regarding fibre orientation is contained in $\langle\boldsymbol{p p p p}\rangle$, which is referred to as the fourth-order moment of the fibre orientation distribution function. This quantity is approximated by solving the equation of change for the second-order moment $\langle p p\rangle$ :

$$
\frac{\mathrm{D}\langle\boldsymbol{p} \boldsymbol{p}\rangle}{\mathrm{D} t}-\nabla \boldsymbol{u}^{T} \cdot\langle\boldsymbol{p} \boldsymbol{p}\rangle-\langle\boldsymbol{p} \boldsymbol{p}\rangle \cdot \nabla \boldsymbol{u}+2 \nabla \boldsymbol{u}:\langle\boldsymbol{p p p p}\rangle=\mathbf{s},
$$

and applying a closure relation to express $\langle\boldsymbol{p p p p}\rangle$ in terms of $\langle\boldsymbol{p p}\rangle$. Equation (2.1e) states that the fibres rotate as material lines, while being advected by the fluid velocity field. The term $2 \nabla \boldsymbol{u}:\langle\boldsymbol{p p p p}\rangle$ ensures that the fibres maintain a constant length, and would have been absent if the fibres could stretch freely, like material lines. Owing to viscosity, there is a no-slip condition on the fibre surface. This induces a resistance against the deformation of the surrounding fluid, which is expressed by the fibre stress tensor $(2.1 c)$.

We express $\langle\boldsymbol{p p p p}\rangle$ in terms of $\langle\boldsymbol{p p}\rangle$ by using the scheme developed by Wetzel (1999). This model relates the principal values of $\langle\boldsymbol{p p p p}\rangle$ to the principal values of $\langle\boldsymbol{p} \boldsymbol{p}\rangle$ by means of a fit to exact solutions. The fit coefficients are constrained to reproduce the correct $\langle\boldsymbol{p p p p}\rangle$ for the three limiting cases of isotropic, bi-axial and uni-axial distribution functions. The subgrid term $\boldsymbol{s}$ is due to the unresolved spatial variations (Gillissen et al. 2007b). This term is modelled as diffusion:

$$
\mathbf{s}=\beta \nabla^{2}\langle\boldsymbol{p p}\rangle,
$$

where $\beta$ is referred to as the artificial diffusivity. The errors in fibre stress in turbulent channel flow due to the subgrid model and the fourth-order moment closure have been shown to be small (Gillissen et al. 2007a, $b$ ).

It is noted that these constitutive equations are valid in the dilute regime $n l^{3} \ll 1$ (Doi \& Edwards 1986). In this limit, fibre stress is negligible compared to Newtonian stress, i.e. the solution behaves as Newtonian and drag reduction does not occur. Significant drag reduction requires $n l^{3} \gg 1$, which is outside the range of the validity of the model. It will be shown that despite this inconsistency the equations are capable of correctly reproducing experimental drag reduction data.

\section{Numerical method}

\subsection{Channel flow}

We simulate turbulent fibre solution channel flow (equation (2.1)). The flow is driven by a constant pressure gradient between two parallel no-slip walls separated by a distance $D$. Periodic boundary conditions are imposed in the homogeneous directions $x$ and $z$. We use a pseudo-spectral flow solver. Spatial derivatives are computed with a Fourier basis for the homogeneous directions and a second-order central finitedifferences scheme for the wall-normal direction $y$. Time integration is achieved with the second-order explicit Adams-Bashforth scheme. Conservation of mass is ensured by using a projection method. The Poisson equation is transformed to Fourier space in the homogeneous directions and a tri-diagonal solver is used for the resulting matrix equations. The variables are discretized on a non-equidistant, staggered mesh. Fibre 


\begin{tabular}{cccrccccccccc}
\hline Run & $R e_{\tau}$ & $R e \times 10^{-3}$ & $\alpha$ & $L_{x}$ & $L_{z}$ & $N_{y}$ & $\beta^{+}$ & $\Delta t^{+}$ & $D R$ & $\delta_{E}^{+}$ & $\epsilon$ & Symbol \\
1 & 360 & 5.7 & 0 & 3 & 1.5 & 128 & 1 & 0.036 & 0 & 0.0076 & - & $\times$ \\
2 & 360 & 6.8 & 25 & 4.5 & 2.25 & 96 & 1.5 & 0.036 & 0.31 & 5.39 & 0.001 & $\square$ \\
3 & 360 & 7.7 & 50 & 4.5 & 2.25 & 96 & 1.5 & 0.036 & 0.45 & 10.9 & 0.008 & $\nabla$ \\
4 & 360 & 8.7 & 100 & 6 & 3 & 96 & 2 & 0.036 & 0.57 & 20.6 & 0.04 & $\square$ \\
5 & 1000 & 18 & 0 & 1 & 0.5 & 384 & 1 & 0.05 & 0 & 0.054 & - & + \\
6 & 1000 & 25 & 50 & 2 & 1 & 192 & 2 & 0.05 & 0.44 & 11.7 & 0.06 & $\nabla$ \\
7 & 1000 & 28 & 100 & 3 & 1.5 & 192 & 4 & 0.01 & 0.55 & 21.4 & 0.006 & $\square$ \\
8 & 1000 & 34 & 300 & 6 & 3 & 96 & 4 & 0.01 & 0.71 & 60.3 & 0.02 & $\bigcirc$ \\
9 & 3000 & 115 & 300 & 2 & 1 & 288 & 6 & 0.06 & 0.69 & 65.1 & 0.008 & $\bigcirc$
\end{tabular}

TABLE 1. Parameters used in the simulations. $R e_{\tau}$ is the frictional Reynolds number, $R e$ is the bulk Reynolds number, $\alpha$ is the concentration parameter, $(2.1 d), L_{x}$ is the ratio of the streamwise and wall-normal channel dimensions, $L_{z}$ is the ratio of the spanwise and wall-normal channel dimensions, $N_{y}$ is the number of grid points in the wall-normal direction, $\beta$ is the artificial diffusivity, $(2.1 f), \Delta t$ is the computational time step, $D R$ is drag reduction, (4.4), $\delta_{E}^{+}$is the elastic layer, (1.1), $\epsilon$ is the relative difference between simulated $D R$ and model (4.6) and the symbols corresponds to those used in figures $1(b), 2(b)$ and 3.

stress, pressure and the velocity components in the homogeneous directions are defined at the cell centres. The wall-normal velocity component is defined on the cell faces.

The usual boundary conditions applied to polymer moment equations are zero wall-normal derivatives at the walls:

$$
\left.\frac{\partial}{\partial y}\langle\boldsymbol{p} \boldsymbol{p}\rangle\right|_{y=0}=\mathbf{0} .
$$

Gillissen et al. (2007b) showed that with these conditions $(2.1 e)$ and $(2.1 f)$ yield $p_{y}(y=0) \neq 0$, resulting in large stress errors in the viscous layer. To obtain $p_{y}(y=0)=0$ the following boundary conditions are employed:

$$
\begin{array}{ll}
\left.(\partial / \partial y)\left\langle p_{i} p_{j}\right\rangle\right|_{y=0}=0 & \text { if } i \neq 2 \text { and } j \neq 2, \\
\left.\left\langle p_{i} p_{j}\right\rangle\right|_{y=0}=0 & \text { if } i=2 \text { or } j=2 .
\end{array}
$$

For these conditions the subgrid term $\beta \nabla^{2}\langle\boldsymbol{p p}\rangle(2.1 f)$ may induce a particle flux through the walls. To conserve the number of fibres we force $\operatorname{tr}\langle\boldsymbol{p} \boldsymbol{p}\rangle=1$ on each grid point and at each time step.

\subsection{Parameters}

The flow is characterized by two dimensionless groups, the frictional Reynolds number $R e_{\tau}$ (1.2) and the concentration parameter $\alpha(2.1 d)$. To determine the friction factor dependence on these parameters, nine runs were carried out using different $R e_{\tau}$ and $\alpha$. Details are given in table 1 . The frictional Reynolds number is based on the friction velocity $U_{\tau}$, which in channel flow is given by: $U_{\tau}=(-(\mathrm{d} \bar{\Pi} / \mathrm{d} x)(D / \rho)(1 / 2))^{1 / 2}$. The channel dimensions and resolutions in $x$ (streamwise), $y$ (wall-normal) and $z$ (spanwise) are $D\left(L_{x} \times 1 \times L_{z}\right)$ and $96 \times N_{y} \times 96$. For the Newtonian flows (runs 1 and 5), the streamwise and spanwise dimensions are approximately 1000 and 500 wall units, which is sufficient to capture the near-wall vortical structures (Jiménez \& Pinelli 1999). These structures increase in size with increasing drag reduction (Li, Sureshkumar \& Khomami 2006). To capture these increasingly large structures the channel dimensions were adapted accordingly. The artificial diffusivity $\beta$ is chosen as small as possible while maintaining a smooth numerical solution to $(2.1 e)$. The grid 


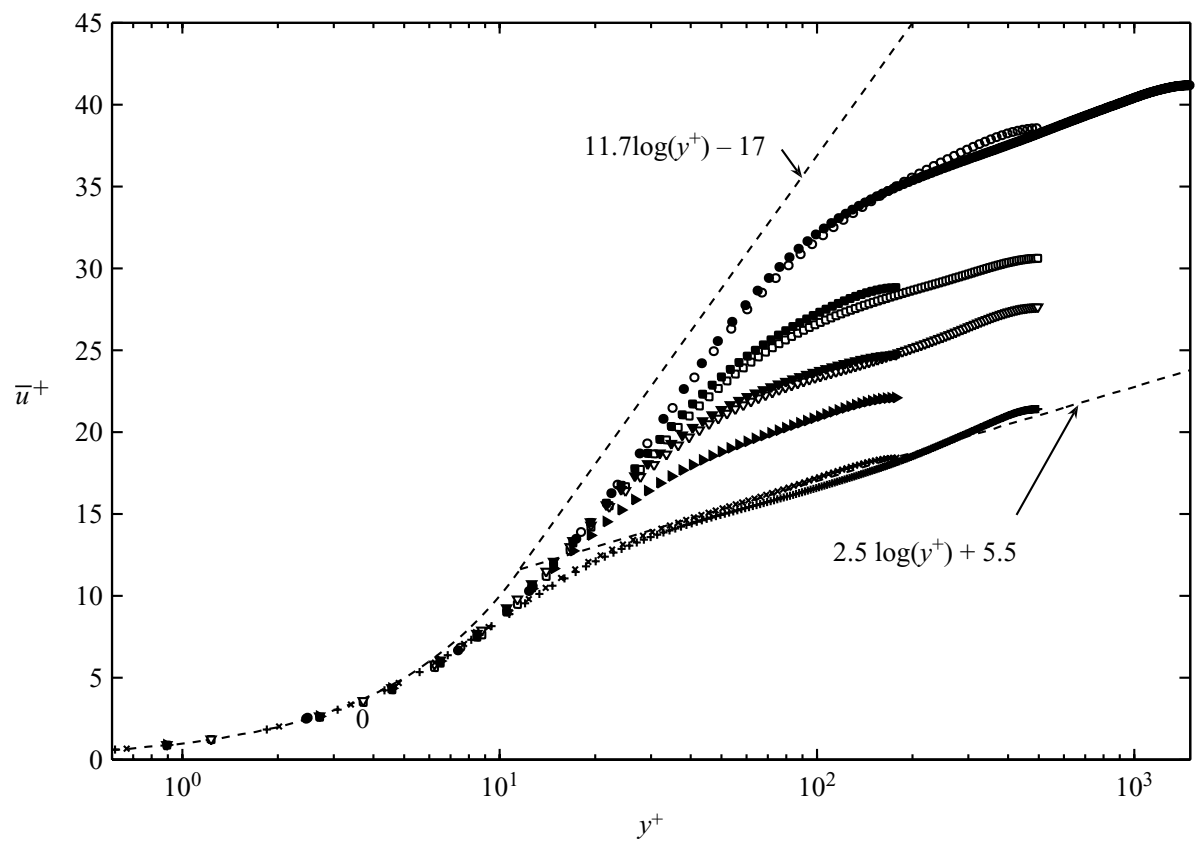

FIGURE 3. Velocity profiles in channel flow simulations. Symbols correspond to parameters listed in table 1.

is non-uniform in the $y$-direction such that $y$ of the $i$ th grid-point is given by

$$
y_{i}=\frac{D}{2}\left[1+\frac{\arctan \left(3 i / N_{y}-\frac{3}{2}\right)}{\arctan \left(\frac{3}{2}\right)}\right] .
$$

\section{Results}

The simulation results are presented in figure 3 , showing the velocity profiles for all runs. The Newtonian profiles (runs 1 and 5) are described well by (1.1) with $\delta_{E}^{+}=0$, i.e. linear in the viscous layer and logarithmic with slope 2.5 in the inertial layer. Also, the non-Newtonian profiles (runs 2-4 and 6-9) are in good agreement with 1.1. Drag reduction is accompanied by a parallel shift of the inertial layer and the elastic layer is approximately logarithmic with slope 11.7. The offset appears to be somewhat smaller than the empirical value of -17 . Another important observation regarding these profiles is illustrated in figure $1(b)$, showing the simulated bulk properties in PK-coordinates. The friction factors are obtained by averaging the non-dimensional velocity profiles over the channel cross-section:

$$
\frac{1}{\sqrt{f}}=\frac{2}{R e_{\tau}} \int_{0}^{R e_{\tau} / 2} \bar{u}^{+} \mathrm{d} y^{+}
$$

Figure 1(b) also shows the empirical parameterization for channel flow, which is obtained by inserting (1.1) into (4.1). It appears that when $\alpha$ is fixed, $\delta_{E}^{+}$is independent of $\operatorname{Re} \sqrt{f}$, which agrees with the experimental evidence presented in figure $1(a)$. The lack of an $\operatorname{Re} \sqrt{f}$ dependence implies that the friction data can be fully parameterized by relating $\delta_{E}^{+}$to $\alpha$, which is done in figure $2(b)$. The $\delta_{E}^{+}$-values are obtained from solving the equation between the simulated $1 / \sqrt{f}$ and the parameterized $1 / \sqrt{f}$, where the latter is obtained by inserting (1.1) into (4.1). A linear relationship can be seen to 


$\begin{array}{llcccc}\text { Source } & \text { Polymer } & M_{w} & \delta_{E}^{+} / c_{m} & l^{*} & l \\ \text { Sasaki (1991b) } & \text { PAA-Na } & 1.7 & 1.4 & 5.1 \pm 0.7 & 5.8 \\ & & 2.4 & 4.4 & 7.4 \pm 1.0 & 8.5 \\ \text { Virk (1975) } & \text { PAMH } & 15 & 2.1 & 52 & 14 \\ \text { Virk et al. (1997) } & & 20 & 5.1 & 70 & 20\end{array}$

TABLE 2. Characterization of rigid polymers used in experimental studies. Distilled water was always used as solvent. Polymer abbreviations are: PAA-Na (Na-polyacrylate) and PAHM (partially hydrolyzed polyacrylamide). $M_{w}$ is the molecular weight given in $\mathrm{Mg} \mathrm{mol}^{-1}$. The values for $\delta_{F}^{+} / c_{m}$ are obtained from figure $2(a)$ and given in millions. The hydraulic length $l$ is obtained from (4.3). The contour length $l^{*}=a M_{w} / M_{a}$, where $M_{a}$ is the molecular weight of the repeat unit and $a$ is the length of the repeat unit, $a=2 \times(1.5 \AA) \cos 35^{\circ}=2.5 \AA$ (Flory 1969). For PAMH: $M_{a}=72 \mathrm{~g} \mathrm{~mol}^{-1}$ and for PAA-Na: $M_{a}=(83 \pm 11) \mathrm{g} \mathrm{mol}^{-1}$. Both $l$ and $l^{*}$ are given in $\mu \mathrm{m}$.

accurately describe the data:

$$
\delta_{E}^{+} \approx 0.2 \alpha \approx 0.02 n l^{3} .
$$

The linear dependence agrees with the experimental data presented in figure 2(a), which further confirms that our numerical model is able to predict the correct physics. This suggest that the rheology of turbulent fibre solutions at large Reynolds number can be described by constitutive equations, which are based on the assumption of diluteness.

Rigid polymers exist in many varieties. The chemical structures have a great impact on their hydraulic properties and (4.2) is not expected to be applicable to all of them. To understand which polymers can be considered hydraulically rod-like requires detailed chemical and rheological analysis, which is outside the scope of the present work. We restrict ourselves to comparing our principle result (4.2) to available experimental data. Using $n l^{3}=c_{m} \rho l^{3} N_{A} / M_{w}$, (4.2) can be written as

$$
\frac{\delta_{E}^{+}}{c_{m}} \approx \frac{0.02 \rho l^{3} N_{A}}{M_{w}} .
$$

The comparison goes as follows. Experimental values for $\delta_{E}^{+} / c_{m}$ are extracted from figure 2(a). After inserting $\delta_{E}^{+} / c_{m}$ into (4.3), we obtain the hydraulic length $l$, which is basically the fibre length for which our numerical model produces the corresponding experimentally measured $\delta_{E}^{+} / c_{m}$. To determine the agreement between our model and the experiment, we compare $l$ to the contour length $l^{*}$, the physical length of the polymer used in the experiment. Unfortunately the available experimental data are very sparse. Only three papers seem to present the required information, given in table 2. For the experiments of Sasaki (1991b), the agreement is nearly perfect, which suggests that these polymers behave hydraulically as rods with $l \approx l^{*}$. Discrepancies for the other experiments might be due to a more complex chemical structure, resulting in a more detailed dependence of $l$ on molecular properties.

Equations (4.5) and (4.2) comprise a complete description of fibre-induced drag reduction. The exact pre-factor in (4.2) is expected to depend on specific chemical properties or effects of non-diluteness and must be considered as an order-ofmagnitude estimate. The elastic layer thickness is a rather abstract concept. More practical is the relative decrease of the friction factor:

$$
D R=1-\frac{f^{P}}{f^{N}},
$$


where the polymer solution $f^{P}$ and in the Newtonian fluid $f^{N}$ are evaluated at the same frictional Reynolds number $R e_{\tau}$. Physically, $D R$ measures the relative increase of the squared bulk velocity, while maintaining a constant pressure drop over the channel. To arrive at an expression for the friction factors, we insert (1.1) into (4.1). In the limit $R e_{\tau} \rightarrow \infty$ this yields

$$
\frac{1}{\sqrt{f}}=2.5 \log R e_{\tau}+9.2 \log \left(1+0.086 \delta_{E}^{+}\right)+1.2 .
$$

Expressions for $f^{P}$ and $f^{N}$ are obtained by substituting $\delta_{E}^{+}=0$ and $\delta_{E}^{+}=0.02 n l^{3}$ respectively. We finally obtain

$$
D R \approx 1-\left(1+\frac{9.2 \log \left(1+0.0017 n l^{3}\right)}{2.5 \log R e_{\tau}+1.2}\right)^{-2} .
$$

The result for pipe flow is similar, with 1.2 replaced by 0 . The relative difference $\epsilon$ between (4.6) and the simulated $D R$-values is listed in table 1. The rather low values reflect the good quality of this relation.

\section{Discussion and conclusion}

We have conducted DNS of turbulent drag reduction in fibre solutions. In the numerical model, interactions between fibres are neglected, conflicting with realistic conditions. To study the implications of this non-physical assumption, the simulations are compared to experimental data from the literature. It is shown that the simulations accurately reproduce three crucial physical observations: (i) the velocity profiles are well described by the empirical parameterization proposed by Virk (1971); (ii) the drag reduction efficiency, measured by the elastic layer $\delta_{E}^{+}$is independent of the frictional Reynolds number and (iii) increases linearly with fibre mass fraction.

From the agreement it is inferred that the rheology in drag-reduced fibre solutions can be described accurately by the dilute equations and the relevant parameter to describe drag reduction is $n l^{3}$. In this regard, the simulation results can be summarized by a linear relationship between $\delta_{E}^{+}$and $n l^{3}$.

The problem of predicting the friction factor for a given fibre solution now becomes that of determining the fibre hydraulic length $l$. To relate this quantity to easily accessible input data requires detailed knowledge on the molecular structure. Extensive experimental work combining turbulent friction analysis and chemical characterization is therefore needed to determine the full implications of the present work.

The research has been partially supported through the PETROMAKS programme funded by The Research Council of Norway. Software to implement the fourthorder moment closure was provided by Professor Charles Tucker of the University of Illinois. The interesting discussions with professor Theo Odijk are greatly appreciated.

\section{REFERENCES}

Benzi, R, De Angelis, E., V. L’vov, V. S. \& Procaccia, I. 2005 Identification and calculation of the universal asymptote for drag reduction by polymers in wall bounded turbulence. Phys. Rev. Lett. 95, 194502.

BEWERSDORFF, H. W. \& SingH, R. P. 1988 Rheological and drag reduction characteristics of xanthan gum solutions. Rheol. Acta 27, 617-627. 
DoI, M. \& Edwards, S. F. 1986 The Theory of Polymer Dynamics. Clarendon.

FLORY, J. 1969 Statistical Mechanics of Chain Molecules. Interscience.

Gillissen, J. J. J., Boersma, B. J., Mortensen, P. H. \& Andersson, H. I. $2007 a$ On the performance of the moment approximation for the numerical computation of fibre stress in turbulent channel flow. Phys. Fluids 19, 035102.

Gillissen, J. J. J., Boersma, B. J., Mortensen, P. H. \& Andersson, H. I. $2007 b$ The stress generated by non-Brownian fibres in turbulent channel flow simulations. Phys. Fluids 19, 115107.

Gyr, A. \& Bewersdorff, H. W. 1995 Drag Reduction of Turbulent Flows by Additives. Kluwer.

JimÉneZ, J. \& Pinelli, A. 1999 The autonomous cycle of near-wall turbulence. J. Fluid Mech. 389, 335-359.

Li, C. F., Sureshrumar, R. \& Khomami, B. 2006 Influence of rheological parameters on polymer induced turbulent drag reduction. J. Non-Newtonian Fluid Mech. 140, 23-40.

Lumley, J. L. 1973 Drag reduction in turbulent flow by polymer additives. J. Polym. Sci. 7, 263-290.

Manhart, M. 2003 Rheology of suspension of rigid-rod like particles in turbulent channel flow. J. Non-Newtonian Fluid Mech. 112, 269-293.

Mortensen, P. H., Andersson, H. I., Gillissen, J. J. J. \& Boersma, B. J. 2008 On the orientation of prolate ellipsoidal particles in a turbulent shear flow. Intl J. Multiphase Flow. (to appear).

RYskin, G. 1987 Turbulent drag reduction by polymers: A quantitative theory. Phys. Rev. Lett. 59, 2059-2062.

SASAKI, S 1991a Drag reduction effect of rod-like polymer solutions. I. Influences of polymer concentration and rigidity of skeletal back bone. J. Phys. Soc. 60, 868-878.

SASAKI, S $1991 b$ Drag reduction effect of rod-like polymer solutions. II. Comparison between microgel and linear type polyions. J. Phys. Soc. 60, 2613-2618.

Sreenivasan, K. R. \& White, C. M. 2000 The onset of drag reduction by dilute polymer additives, and the maximum drag reduction asymptote. J. Fluid Mech. 409, 149-164.

Tennekes, H. \& Lumley, J., L. 1973 A First Course in Turbulence. MIT Press.

VIRK, P. S. 1971 An elastic sublayer model for drag reduction by dilute solutions of linear macromolecules. J. Fluid Mech. 45, 417-440.

VIRK, P. S. 1975 Drag reduction by collapsed and extended polyelectrolytes. Nature 253, 109-110.

Virk, P. S., Sherman, D. C. \& WaGger, D. L. 1997 Additive equivalence during turbulent drag reduction. AIChE J. 43, 3257-3259.

WeTZEL, E. D. 1999 Modeling flow-induced microstructure in inhomogeneous liquid-liquid mixtures. $\mathrm{PhD}$ thesis, University of Illinois at Urbana-Champaign, Urbana, IL. 